Pacific Journal of Mathematics

MULTI-TUPLE HULLS 


\title{
MULTI-TUPLE HULLS
}

\author{
TOMA V. TONEV
}

\begin{abstract}
We study two general families of hulls related to vector-valued functions and their interrelations with multi-tuple Shilov boundaries of uniform algebras.
\end{abstract}

1. Introduction. The classical hulls-polynomial, rational, holomorphic, $A$-convex etc. are tightly and naturally connected with functional approximations and interpolations. Recently several new families of hulls have come into appearance. Namely Basener [2] has used a generalization of the family of polynomial hulls in his study of $q$-holomorphic functions. Recently Slodkowski [8] has used a generalization of rational hulls in his investigation of analytic perturbation of Taylor spectrum, and Corach and Suárez [3] have introduced a general family of rational hulls in order to evaluate the topological stable rank of some algebras. In [11] there were investigated the properties of two families of hulls, the so called $n$-tuple rational A-convex hulls and $n$-tuple A-convex hulls.

The multi-tuple Shilov boundaries of commutative Banach algebras have proved to be essential tools in the investigation of multidimensional analytic structures in algebra spectra. Results concerning relationships between these boundaries and the analyticity in algebra spectra have appeared often during the last fifteen years (e.g. Basener [1], Kumagai [5], Sibony [6], Tonev ([11], [12]) etc.). Various properties of multi-dimensional Shilov boundaries have been investigated by Basener [1], [2], Sibony [6], Slodkowski ([7], [8]), Tonev ([10], [11]) and others.

In this paper we establish a unified approach to the above mentioned families of hulls, study their properties and investigate their interrelations with the vector valued functions and multi-tuple Shilov boundaries of uniform algebras.

2. Multi-tuple rational $A$-convex hulls. Let $A$ be a uniform algebra over $\mathbf{C}$ with unit. That is $A$ is a separating closed subalgebra of the space of all continuous complex valued functions on some compact Hausdorff space $X$ which contains the constants and the norm of 
$f \in A$ is the maximum of $|f(x)|$ on $X$. As usual $\operatorname{sp} A$ denotes the maximal ideal space of $A$ and $\hat{f}$ denotes the Gelfand extension of a given function $f$ of $A$. The Shilov boundary $\partial A$ of $A$ is the smallest closed subset of $\operatorname{sp} A$ on which the Gelfand extensions of all functions of $A$ assume the maximums of their absolute values. Throughout this paper we shall assume that $\operatorname{sp} A$ is identified with the set $X$ and that the Gelfand extensions $\hat{f}(m)$ are identified with the algebra elements $f(m)$. $A^{n}$ will denote the set of all $n$-tuples of functions from $A$.

Definition 1. The $n$-tuple rational $A$-convex hull $r_{n}(E)$ of a subset $E$ of $\operatorname{sp} A$ is the biggest among all closed subsets $K$ of $\operatorname{sp} A$ for which the equality

$$
\min _{x \in K}\|F(x)\|=\min _{x \in E}\|F(x)\|
$$

holds for every $n$-tuple $F=\left(f_{1}, \ldots, f_{n}\right)$ of functions in $A . E$ is called $n$-tuple rationally $A$-convex if $r_{n}(E)=E$.

Obviously $r_{n}(E)$ is a closed subset of $\operatorname{sp} A$. One can see that $r_{n}(E)=\left\{m \in \operatorname{sp} A:\|S(m)\| \geq \min _{m \in E}\|S(m)\|\right.$ for every $S \subset A$ with $\# S \leq n\}$. Naturally, the last inequality is essential for regular subsets $S$ of $A$ only. As a corollary from this observation we get that $E \subset \cdots \subset r_{n+1}(E) \subset r_{n}(E) \subset \cdots \subset r_{1}(E) \subset \operatorname{sp} A$.

The next proposition gives a useful characterization of the hulls $r_{n}(E)$.

Proposition 1. The $n$-tuple rational $A$-convex hull of a subset $E$ of $\operatorname{sp} A$ coincides with the set $r_{n}(E)=\{m \in \operatorname{sp} A: F(m) \in F(E)$ for all $\left.F \in A^{n}\right\}$.

Proof. Denote for a while the set $\{m \in \operatorname{sp} A: F(m) \in F(E)$ for all $\left.F \in A^{n}\right\}$ by $K$. Let $m_{0} \in r_{n}(E)$ and let $F \in A^{n}$ be such that $F\left(m_{0}\right)$ $=0$. By (1) $F$ vanishes within $E$ so that $0=F\left(m_{0}\right) \in F(E)$. Hence $K \supset r_{n}(E)$. Assuming conversely that $K \backslash r_{n}(E) \neq \varnothing$, for any point $m_{0} \in K \backslash r_{n}(E)$ we have $\left\|F\left(m_{0}\right)\right\|<\min _{m \in E}\|F(m)\|$ for some $F \in A^{n}$. Hence $H\left(m_{0}\right)=0$ but $0 \notin H(E)$ for $H=F-F\left(m_{0}\right) \in A^{n}$ in contradiction with $m_{0} \in K$. The proposition is proved.

The $n$-tuple rational $A$-convex hull is a multi-tuple version of the rational A-convex hull $r(E)=\{m \in \operatorname{sp} A: f(m) \in f(E)$ for all $f \in A\}$ of any subset $E$ of the spectrum of a uniform algebra $A$-namely, as it is easy to check, the 1-tuple rational $A$-convex hull $r_{1}(E)$ coincides with $r(E)$. 
From Proposition 1 it follows that the $n$-tuple rational $A$-convex hulls coincide with the sets $\bigcap_{F \in A^{n}} F^{-1} \circ F(E)$, i.e. with the generalized rational hulls introduced by Corach and Suárez in [3] and utilized by them in their recent investigations on topological stable ranks of commutative Banach algebras.

Since $F(m) \in F(E)$ if and only if the function $H=F-F(m)$ vanishes on $E$, Proposition 1 implies the following

Proposition 2. The n-tuple rational A-convex hull $r_{n}(E)$ of a closed subset $E$ of $\operatorname{sp} A$ coincides with the set of these points $m$ in $\operatorname{sp} A$ such that every $n$-tuple $F \in A^{n}$ with $F(m)=0$ vanishes on $E$.

In general the $n$-tuple rational $A$-convex hull of a subset of $\operatorname{sp} A$ does not coincide with the algebra spectrum. For instance if $A$ is the disc-algebra $A(\Delta)$, then $r_{1}\left(S^{1}\right)=r_{1}(\partial A)=S^{1} \neq \bar{\Delta}=\operatorname{sp} A$, as one can see by applying, say, (1) to the identity function in $\mathbf{C}^{1}$. In this respect the following corollary from Proposition 2 is of some interest.

CoRollary 1 [3]. $r_{n}(E)=\operatorname{sp} A$ if and only if every $n$-tuple $F(m)$ over $A$ which does not vanish on $E$ is regular.

EXAMPLE 1. If $n \geq 2$, then the 1-tuple rational $A\left(B^{n}(1)\right)$-convex hull of the unit sphere $S^{n}(1)$ in $\mathbf{C}^{n}$ is the unit ball $B^{n}(1)$.

Indeed, as known, if $n \geq 2$ any holomorphic function vanishes on $S^{n}(1)$ whenever it vanishes inside $B^{n}(1)$.

EXAMPLE 2. The $n$-tuple rational convex hulls $\rho_{n}(E)$.

Let $\Lambda$ be an arbitrary set and let $\mathbf{C}^{\Lambda}$ be the Cartesian product of $\Lambda$ copies of the complex plane, equipped by the natural topology. Given a compact subset $E$ in $\mathbf{C}^{\Lambda}$ let $R(E)$ be the closure in $C(E)$ of all rational functions $p / q$ in $\mathbf{C}^{\Lambda}$ with non-vanishing on $E$ denominators $q$. Since these rational functions are dense in $R(E)$, the $n$-tuple rational $R(E)$-convex hull of $E$ is the biggest among all compact subsets $N$ of $\mathbf{C}^{\Lambda}$, such that $\left(r_{1}, \ldots, r_{n}\right)(N)=\left(r_{1}, \ldots, r_{n}\right)(E)$ for every $n$-tuple $\left(r_{1}, \ldots, r_{n}\right)$ of rational functions $r_{j}=p_{j} / q_{j}$ in $\mathbf{C}^{\Lambda}$ with $q_{j} \neq 0$ on $N$. We shall refer to this hull as $n$-tuple rational convex hull of $E$ and shall denote it by $\rho_{n}(E)$.

Being the $n$-tuple rational $R(E)$-convex hull of $E, \rho_{n}(E)$ is the biggest among all sets $N$ in $\mathbf{C}^{\Lambda}$ for which the inequality

$$
\inf _{\mathbf{z} \in N}\left\|\left(r_{1}(\mathbf{z}), \ldots, r_{n}(\mathbf{z})\right)\right\|=\min _{\mathbf{z} \in E}\left\|\left(r_{1}(\mathbf{z}), \ldots, r_{n}(\mathbf{z})\right)\right\|
$$


holds for every $n$-tuple $\left(r_{1}, \ldots, r_{n}\right)$ of rational functions in $\mathbf{C}^{\Lambda}$ with non-vanishing on $E$ denominators.

A subset $E \subset \mathbf{C}^{\Lambda}$ is $n$-tuple rationally convex if $\rho_{n}(E)=E$. The $n$-tuple rational convex hulls $\rho_{n}(E)$ are natural generalizations of the usual rational convex hulls $r(E)=\left\{\mathbf{z} \in \mathbf{C}^{\Lambda}:|r(\mathbf{z})| \leq \max _{\mathbf{z} \in E}|r(\mathbf{z})|\right.$ for every rational function $r(\mathbf{z})$ that is bounded on $E$-namely by Corollary 1 one can observe that the 1-tuple rational convex hull $\rho_{1}(E)$ of a set $E \subset C^{\Lambda}$ coincides with $r(E)$.

By applying the relation from Proposition 1 to the identity mapping in $\mathbf{C}^{n}$ we get

Proposition 3. Every compact set $E$ in $\mathbf{C}^{n}$ is $k$-tuple rationally convex for any $k \geq n$.

As we shall see below, $\rho_{n}(E)$ coincides with the $n$-tuple rational $P(E)$-convex hull of $E$, i.e. the $n$-tuple rational $A$-convex hulls of all compact subsets $E$ in $\mathbf{C}^{\Lambda}$ are equal for both algebras $A=P(E)$ and $A=R(E)$.

PROPOSITION 4. The $n$-tuple rational hull $\rho_{n}(E)$ of every compact subset $E$ in $\mathbf{C}^{\Lambda}$ is equal to its $n$-tuple rational $P(E)$-convex hull.

Proof. Denote for a while the $n$-tuple rational $A$-convex hull of a set by $r_{n}^{A}(E)$. Clearly $r_{n}^{P(E)}(E) \supset r_{n}^{R(E)}(E)=\rho_{n}(E)$, because $P(E) \subset R(E)$. If we assume that $r_{n}^{P(E)}(E) \backslash \rho_{n}(E) \neq \varnothing$, by Proposition 3 we can find a point $m_{0}$ in $r_{n}^{P(E)}(E)$ and an $n$-tuple $F \in$ $R^{n}(E)$ such that $F\left(m_{0}\right)=0$, but $\|F(m)\| \neq 0$ on $E$. If $F=$ $\left(f_{1}, \ldots, f_{n}\right), f_{j}=p_{j} / q_{j}$, where $p_{j}, q_{j}$ are polynomials, $q_{j} \neq 0$ on $E$, then $\left(p_{1}\left(m_{0}\right), \ldots, p_{n}\left(m_{0}\right)\right)=0$ but the $n$-tuple $\left(p_{1}, \ldots, p_{n}\right)$ does not vanish on $E$. Proposition 3 indicates that this contradicts the choice of the point $m_{0} \in r_{n}^{P(E)}(E)$.

In the case $n=1$ Proposition 4 restricts to the well known equality between the rational hull and the $P(E)$-convex hull of a set $E \in \operatorname{sp} A$, i.e. $r(E)=h^{P(E)}(E)$ (e.g. [4]), which by the way motivates the names " $n$-tuple rational hull" and " $n$-tuple rational $A$-convex hull" given to the sets $\rho_{n}(E)$ and $r_{n}^{A}(E)$ respectively.

Together with Proposition 2, Proposition 4 implies the following

COROLlARY 2. The $n$-tuple rational convex hull $\rho_{n}(E)$ of a compact subset $E$ of $\mathbf{C}^{\Lambda}$ coincides with the set of all points $\mathbf{z} \in \mathbf{C}^{\Lambda}$ such that for 
every $n$-tuple of polynomials $\left(p_{1}, \ldots, p_{n}\right)$ vanishing at $\mathbf{z}$ the variety $\left\{\mathbf{y} \in \mathbf{C}^{\Lambda}: p_{j}(\mathbf{y})=0, j=1, \ldots, n\right\}$ meets $E$.

The sets described in Corollary 2 are precisely the $(n-1)$-th rational hulls which were introduced by Slodkowski in [8] and used by him in his recent investigation of analytic perturbation of Taylor spectrum of $n$-tuples of commuting operators. Note that, as shown in [3], $\bigcap_{n \geq 1} r_{n}(E)=E$.

Let $V\left(f_{1}, \ldots, f_{n}\right)$ be the vanishing set of a fixed $n$-tuple $\left(f_{1}, \ldots\right.$, $\left.f_{n}\right)$ over $A$, i.e.

$$
V\left(f_{1}, \ldots, f_{n}\right)=\left\{m \in \operatorname{sp} A: f_{1}(m)=f_{2}(m)=\cdots=f_{n}(m)=0\right\} .
$$

Denote by $A_{E}$ the closure in $C(E)$ of restrictions of all elements of $A$ on a fixed closed subset $E$ of $\operatorname{sp} A$.

THEOREM 1. Let $k$ be a fixed integer, $1 \leq k \leq n-1$. The n-tuple rational A-convex hull $r_{n}(E)$ of a closed subset $E$ of $\operatorname{sp} A$ coincides with the set of these points $m \in \operatorname{sp} A$ which belong to the $k$-tuple rational $A_{V(S)}$-convex hulls $r_{k}(E \cap V(S))$ of the sets $E \cap V(S)$ for every set $S$ in $A$ such that $\# S \leq n-k$ and with $m$ belonging to $V(S)$.

Proof. Let $K=\left\{m \in \operatorname{sp} A: m \in r_{k}(E \cap V(S))\right.$ for every $S \subset A$ with $\# S \leq n-k$ and $m \in V(S)\}$. Suppose that $K \backslash r_{n}(E) \neq \varnothing$ and let $m_{0} \in$ $K \backslash r_{n}(E)$. By Definition 1 we can find an $n$-tuple $F=\left(f_{1}, \ldots, f_{n}\right)$ over $A$ such that $\left\|F\left(m_{0}\right)\right\|<\min _{m \in E}\|F(m)\|$. By applying, if necessary, an orthogonal transformation in $\mathbf{C}^{n}$, we can suppose from the beginning that $F\left(m_{0}\right)=\left(f_{1}\left(m_{0}\right), \ldots, f_{k}\left(m_{0}\right), 0, \ldots, 0\right)$. Hence $m_{0} \in V(S)$ where $S=\left(f_{k+1}, \ldots, f_{n}\right)$. For $T=\left(f_{1}, \ldots, f_{k}\right)$ we have $\left\|T\left(m_{0}\right)\right\|=\left\|F\left(m_{0}\right)\right\|<\min _{m \in E}\|F(m)\| \leq \min _{E \cap V(S)}\|F(m)\|$ $=\min _{E \cap V(S)}\|T(m)\|=\min _{r_{k}(E \cap V(S))}\|T(m)\|$. Consequently $m_{0} \notin$ $r_{k}(E \cap V(S))$ in contradiction with $m_{0} \in K$. We conclude that $K \subset r_{n}(E)$.

Suppose conversely that $r_{n}(E) \backslash K \neq \varnothing$ and let $m_{0} \in r_{n}(E) \backslash K$. Let $S$ be an $(n-k)$-tuple over $A$ such that $m_{0} \in V(S) \backslash r_{k}(E \cap V(S))$. Consequently there exists a $k$-tuple $T \in A_{V(S)}^{k}$, such that $\left\|T\left(m_{0}\right)\right\|<$ $r=\min _{E \cap V(S)}\|T(m)\|$. Without loss of generality we can assume that $T \in A^{k}$. For every positive $\varepsilon<r$ we can find a neighborhood $V_{\varepsilon}$ of the set $E \cap V(S)$ in $E$ on which $\|T(m)\|>r-\varepsilon$. Hence for every $m \in E$ we have

$$
\left(C_{\varepsilon}^{2}\|S(m)\|^{2}+\|T(m)\|^{2}\right)^{1 / 2}>r-\varepsilon
$$


for some positive constant $C_{\varepsilon}$ large enough. Because $\left(C_{\varepsilon} S, T\right) \in$ $A^{n}$, (2) holds also on $r_{n}(E)$. In particular at $m_{0} \in V(S)$ we have $\left\|T\left(m_{0}\right)\right\|>r-\varepsilon$ and henceforth $\left\|T\left(m_{0}\right)\right\| \geq r$ because of the liberty of the choice of $\varepsilon$. Since this contradicts with the initial inequality $\left\|T\left(m_{0}\right)\right\|<r$, we conclude that $r_{n}(E) \subset K$. The theorem is proved.

The case $k=1$ from Theorem 1 in particular says

COROLlaRy 3. The n-tuple rational A-convex hull $r_{n}(E)$ of a closed set $E$ in $\operatorname{sp} A$ coincides with the set of these points $m \in \operatorname{sp} A$ which belong to the rational $A_{V(S)}$-convex hulls $r(E \cap V(S))$ of the sets $E \cap$ $V(S)$ for any set $S$ in $A$ whose cardinality does not exceed $n-1$ and such that $m$ belongs to $V(S)$, i.e.

$$
\begin{array}{r}
r_{n}(E)=\{m \in \operatorname{sp} A: m \in r(E \cap V(S)) \text { for any } S \subset A \text { with } \\
\# S \leq n-1, m \in V(S)\} .
\end{array}
$$

3. Multi-tuple $A$-convex hulls. In this section we introduce another family of hulls in algebra spectra by putting some limitations on the $n$-tuples $F$ from (1). Recall that an $n$-tuple $F=\left(f_{1}, \ldots, f_{n}\right) \in A^{n}$ is called regular if the functions $f_{1}, \ldots, f_{n}$ have no common zeros on $\operatorname{sp} A$, i.e. if $V\left(f_{1}, \ldots, f_{n}\right)=\varnothing$, or in other words, if the mapping $F$ does not vanish on $\operatorname{sp} A$.

Definition 2. The $n$-tuple $A$-convex hull $h_{n}(E)$ of a closed subset $E$ of $\operatorname{sp} A$ is the union of all closed subsets $N$ of $\operatorname{sp} A$ which contain $E$ and such that the inequality

$$
\min _{m \in N}\|F(m)\|=\min _{m \in E}\|F(m)\|
$$

holds for every non-vanishing on $N n$-tuple $F$ of functions from $A$. $E$ is an $n$-tuple $A$-convex set if it coincides with its $n$-tuple $A$-convex hull $h_{n}(E)$.

The $n$-tuple $A$-convex hulls are closed subsets of $\operatorname{sp} A$, since the closure [N] of a set $N \in \operatorname{sp} A$ that satisfies (3) also satisfies (3). In fact $h_{n}(E)$ coincides with the union of all subsets $N$ of $\operatorname{sp} A$ which satisfy (3).

The next proposition, which proof is analogical to that of Proposition 1, gives a useful characterization of the hulls $h_{n}(E)$.

Proposition 5. Let $E$ be a closed subset of $\operatorname{sp} A$. The $n$-tuple $A$ convex hull $h_{n}(E)$ of $E$ coincides with the biggest among all subsets 
$N$ of $\operatorname{sp} A$ which contain $E$ and for which

$$
b F(N) \subset F(E)
$$

for every $F \in A^{n}$.

Proof (see also [11]). First we show that if $N=h_{n}(E)$ then (3) holds for every non-vanishing on $h_{n}(E)$ mapping $F \in A^{n}$. Clearly $0 \notin F(E)$ for every such $F \in A^{n}$. If $c=\min _{m \in E}\|F(m)\|$ then $F(E) \subset \mathbf{C}^{n} \backslash B(c)=\left\{\mathbf{z} \in \mathbf{C}^{n}:\|\mathbf{z}\| \geq c\right\}$ and by the definition of $h_{n}(E)$ we have that $b F\left(h_{n}(E)\right) \subset \mathbf{C}^{n} \backslash B(c)$. This implies $F\left(h_{n}(E)\right) \subset$ $\mathrm{C}^{n} \backslash B(c)$. Consequently

$$
\min _{m \in h_{n}(E)}\|F(m)\| \geq c=\min _{m \in E}\|F(m)\| .
$$

Since the opposite inequality is obviously fulfilled, we conclude that (3) holds with $N=h_{n}(E)$ for every $F \in A^{n}$ with $\|F(m)\| \neq 0$ on $h_{n}(E)$.

Let now $N$ be a closed subset of $\operatorname{sp} A$ which satisfies (3) for every $F \in A^{n}$ that does not vanish on $N$, and assume that (4) is false, i.e. that $b F(N) \backslash F(E) \neq \varnothing$. Let $\mathbf{z}_{0}$ be a point from $b F(N) \backslash F(E)$ and let $m_{0} \in F^{-1}\left(\mathbf{z}_{0}\right)$. Obviously

$$
\left\|H\left(m_{0}\right)\right\|<\min _{m \in E}\|H(m)\|
$$

for the $n$-tuple $H=F-\mathbf{z}_{0}$. We can find also a point $\mathbf{z}_{1} \in \mathbf{C}^{n} \backslash F(N)$ close enough to $\mathbf{z}_{0}$, such that (5) holds for the $n$-tuple $H_{1}=F-\mathbf{z}_{1}$. But this contradicts to (3) since obviously $H_{1}(m)$ does not vanish on $N$. The proposition is proved.

As the following example shows, the $n$-tuple $A$-convex hulls $h_{n}(E)$ are natural multi-tuple versions of the A-convex hulls $h(E)=\{m \in$ $\operatorname{sp} A:|f(m)| \leq \max _{m \in E}|F(m)|$ for all $\left.f \in A\right\}$ of closed sets $E$ in $\operatorname{sp} A$. Recall that $h(E)$ consists of all linear multiplicative functionals of $A$ that possess continuous extensions on $A_{E}$. A set $E \in \operatorname{sp} A$ is $A$ convex if $h(E)=E$, i.e. if $E=\left\{m \in \operatorname{sp} A:|f(m)| \leq \max _{m \in E}|f(m)|\right.$ for all $f \in A\}$, or, equivalently, if $\operatorname{sp} A_{E}=E$ (e.g. [4]). The vanishing set $V(S)$ of any subset $S$ of $A$ is a simple example for an $A$-convex set.

EXAMPLE 3. The 1-tuple $A$-convex hull $h_{1}(E)$ of each closed subset $E$ of $\operatorname{sp} A$ coincides with its usual $A$-convex hull $h(E)$. 
Indeed, since $b f\left(h_{1}(E)\right) \subset f(E)$, we have that $\max _{m \in h_{1}(E)}|f(m)|$ $\leq \max _{m \in E}|f(m)|$ for every function $f \in A$ and consequently $h_{1}(E) \subset$ $h(E)$ by the definition of the $A$-convex hull $h(E)$. Assume that $h(E)$ contains properly $h_{1}(E)$. Then there exists a function $f$ from $A$ such that $b f(h(E)) \not \subset f(E)$. Let $m_{0}$ be a point from $h(E)$, such that $f\left(m_{0}\right) \in b f(h(E)) \backslash f(E)$. By choosing a point $z_{0}$ from $\mathbf{C} \backslash f(h(E))$ close enough to $f\left(m_{0}\right)$, we can construct a function $g(z)=f(z)$ $z_{0} \in A_{h(E)}^{-1}$ for which $\left|g\left(m_{0}\right)\right|<\min _{m \in E}|g(m)|$. Hence $1 /\left|g\left(m_{0}\right)\right|>$ $\max _{m \in E} 1 /|g(m)|$. Since $1 / g \in A_{h(E)}$ and $\operatorname{sp} A_{h(E)}=h(E)$, there exists a function $g_{1}$ from $A$ such that $\left|g_{1}\left(m_{0}\right)\right|>\max _{m \in E}\left|g_{1}(m)\right|$, i.e. $m_{0} \notin h(E)$ in contradiction with the choice of $m_{0}$.

In particular we obtain that $h(E)$ is the biggest among all closed subsets $N$ of $\operatorname{sp} A$ such that $b f(N) \subset f(E)$ for every $f \in A$.

EXAMPLE 4. The $n$-tuple polynomial convex hulls $\pi_{n}(E)$.

Given a compact subset $E$ in $\mathbf{C}^{\Lambda}$ denote by $P(E)$ the closure in $C(E)$ of the set of all polynomials in $\mathbf{C}^{\Lambda}$. Since the polynomials are dense in $P(E)$, the $n$-tuple $P(E)$-convex hull of $E$ is the biggest among all closed subsets $N$ of $\mathbf{C}^{\Lambda}$ such that $b\left(p_{1}, \ldots, p_{n}\right)(N) \subset$ $\left(p_{1}, \ldots, p_{n}\right)(E)$ for every $n$-tuple $\left(p_{1}, \ldots, p_{n}\right)$ of polynomials in $\mathrm{C}^{\Lambda}$. We shall refer to this hull as $n$-tuple polynomial convex hull of $E$ and shall denote it by $\pi_{n}(E)$. Clearly $E \subset \rho_{n}(E) \subset \pi_{n}(E)$ for every compact subset $E$ in $\mathbf{C}^{\Lambda}$.

Being the $n$-tuple $P(E)$-convex hull of $E, \pi_{n}(E)$ is the biggest among all sets $N$ in $\mathrm{C}^{\Lambda}$ which contain $E$ and such that the inequality

$$
\inf _{\mathbf{z} \in N}\left\|\left(p_{1}(\mathbf{z}), \ldots, p_{n}(\mathbf{z})\right)\right\|=\min _{\mathbf{z} \in E}\left\|\left(p_{1}(\mathbf{z}), \ldots, p_{n}(\mathbf{z})\right)\right\|
$$

holds for every non-vanishing on $n n$-tuple $\left(p_{1}, \ldots, p_{n}\right)$ of polynomials in $\mathbf{C}^{\Lambda}$.

A subset $E \subset \mathbf{C}^{\Lambda}$ is $n$-tuple polynomially convex if it coincides with its $n$-tuple polynomial hull $\pi_{n}(E)$. By applying the inclusion

$$
b\left(p_{1}, \ldots, p_{n}\right)\left(\pi_{n}(E)\right) \subset\left(p_{1}, \ldots, p_{n}\right)(E)
$$

to the identity mapping in $\mathbf{C}^{n}$ we get that $b\left(\pi_{n}(E)\right) \subset E$ for every compact subset $E$ in $\mathbf{C}^{n}$. This implies that the $n$-tuple polynomial convex hull $\pi_{n}(E)$ of $E$ is contained in the union of $E$ and the union of all bounded components of its complement in $\mathbf{C}^{n}$. Because of its maximality property, $\pi_{n}(E)$ actually coincides with this union. Therefore $E$ is n-tuple polynomially convex if and only if its complement $\mathbf{C}^{n} \backslash E$ does not possess bounded components. 
The $n$-tuple polynomial convex hulls $\pi_{n}(E)$ of subsets $E$ of $\mathbf{C}^{\Lambda}$ are natural generalizations of their usual polynomial hulls $\widehat{E}=\left\{\mathbf{z} \in \mathbf{C}^{\Lambda}\right.$ : $|p(\mathbf{z})| \leq \max _{\mathbf{z} \in E}|p(\mathbf{z})|$ for every polynomial $p$ in $\left.\mathbf{C}^{\Lambda}\right\}$-as Example 3 shows, $\pi_{1}(E)=\widehat{E}$. In general hulls $\pi_{n}(E)$ are different from the usual polynomial hulls $\widehat{E}$. For instance the 2-tuple polynomial hull $\pi_{2}(E)$ of the set $E=\left\{\left(z_{1}, z_{2}\right) \in \mathbf{C}^{2}: 1 \leq\left|z_{1}\right| \leq 2,\left|z_{2}\right|=0\right\} \subset \mathbf{C}^{2}$ is the set $E$ itself, which does not coincide with the usual polynomial hull $\widehat{E}=\left\{\left(z_{1}, z_{2}\right) \in \mathbf{C}^{2}:\left|z_{1}\right| \leq 2,\left|z_{2}\right|=0\right\}$. Indeed, for any $z_{0} \notin E$ we can choose an $\varepsilon>0$ small enough, so that the regular pair of polynomials $\left(z_{1}-z_{0}, z_{2}+\varepsilon\right)$ to attain the minimum of its norm near $z_{0}$ and outside $E$ at the same time. This means that $z_{0} \notin \pi_{2}(E)$ for any $z_{0} \notin E$, i.e. that $\pi_{2}(E) \subset E$ and hence $\pi_{2}(E)=E$ since $\pi_{2} \supset E$ by Definition 2 .

As we know, $r_{n}(E) \subset h_{n}(E)$ for every closed subset $E$ of $\operatorname{sp} A$. The following corollary establishes a somewhat opposite inclusion.

COROLlaRY 4. Let $E$ be a closed subset of the spectrum of a uniform algebra $A$. Then $h_{n}(E) \subset r_{n-1}(E)$.

Proof (see also [11]). If $m_{0} \notin r_{n-1}(E)$, then by Definition 1 we can find an $(n-1)$-tuple $F=\left(f_{1}, \ldots, f_{n-1}\right)$ of functions from $A$ with $\left\|F\left(m_{0}\right)\right\|<\min _{m \in E}\|F(m)\|$. The regular $n$-tuple $\left(f_{1}, \ldots, f_{n}, 1\right)=$ $(F, 1)$ therefore satisfies the inequality

$$
\left\|F\left(m_{0}\right)\right\|^{2}+1<\min _{m \in E}\|F(m)\|^{2}+1 .
$$

Definition 2 indicates that $m_{0} \notin h_{n}(E)$. We conclude that $h_{n}(E) \subset$ $r_{n-1}(E)$, as claimed.

Observe that $F\left(h_{n}(E)\right) \subset \pi_{n}(F(E))$ for any $F \in A^{n}$ because $F(E) \supset$ $b F\left(h_{n}(E)\right)$ and hence $h_{n}(E) \subset \bigcap_{F \in A^{n}} F^{-1}\left(\pi_{n}(F(E))\right)$. In fact both sets are equal. Indeed, denote the latter set by $K$ and take an $F \in A^{n}$ with $\|F(m)\| \neq 0$ on $K$. Clearly $\|\mathbf{z}\| \geq \min _{m \in E}\|F(m)\|$ for every $\mathbf{z} \in \pi_{n}(F(E))$ because $F(m)$ does not vanish on the set $E \subset K$. Thus $\left\|F\left(m_{0}\right)\right\| \geq \min _{m \in E}\|F(m)\|$ for every point $m_{0}$ in $F^{-1}\left(\pi_{n}(F(E))\right)$ and therefore for every point $m_{0}$ in $K$ as well. Hence $h^{n}(E) \supset K$, i.e.

CoRollaRy 5. $h_{n}(E)=\bigcap_{F \in A^{n}} F^{-1}\left(\pi_{n}(F(E))\right)$.

The next theorem is an $n$-tuple $A$-convex analogue of Corollary 3 . Its proof follows the same lines as the proof of the case $k=1$ of Theorem 1. 
THEOREM 2. The n-tuple A-convex hull $h_{n}(E)$ of a closed set $E$ in $\operatorname{sp} A$ coincides with the set of these points $m \in \operatorname{sp} A$ which belong to the $A_{V(S)}$-convex hulls $h(E \cap V(S))$ of the sets $E \cap V(S)$ for any set $S$ in $A$ whose cardinality does not exceed $n-1$ and such that $m$ belongs to $V(S)$, i.e.

$$
\begin{aligned}
& h_{n}(E)=\{m \in \operatorname{sp} A: m \in h(E \cap V(S)) \text { for any } S \subset A \\
& \text { with } \# S \leq n-1, m \in V(S)\} .
\end{aligned}
$$

Proof. Denote for a while the set $\{m \in \operatorname{sp} A: m \in h(E \cap V(S))$ for any $S \subset A$ with $\# S \leq n-1, m \in V(S)\}$ by $K$ and suppose that $K \backslash h_{n}(E) \neq \varnothing$. By Definition 2 we can find an $n$-tuple $F=$ $\left(f_{1}, \ldots, f_{n}\right) \subset A^{n}$ which does not vanish on $K$ and such that $\left\|F\left(m_{0}\right)\right\|<\min _{m \in E}\|F(m)\|$ for some $m_{0} \in K \backslash h_{n}(E)$.

Without loss of generality (applying, if necessary, an orthogonal transformation in $\mathbf{C}^{n}$ ) we can assume from the beginning that $F\left(m_{0}\right)$ $=\left(f_{1}\left(m_{0}\right), 0, \ldots, 0\right)$. Hence $m_{0} \in V(S)$ for $S=\left(f_{2}, \ldots, f_{n}\right)$ and $f_{1}$ does not vanish on $K \cap V(S)$. Then $\left|f_{1}\left(m_{0}\right)\right|=\left\|F\left(m_{0}\right)\right\|<$ $\min _{m \in E}\|F(m)\| \leq \min _{E \cap V(S)}\|F(m)\|=\min _{E \cap V(S)}\left|f_{1}(m)\right|$, i.e. $\max _{m \in E \cap V(S)}|g(m)|<\left|g\left(m_{0}\right)\right|$ for $g=1 / f_{1} \in A_{V(S)}$. Consequently $m_{0} \notin h(E \cap V(S))$ in contradiction with $m_{0} \in K$. We conclude that $K \subset h_{n}(K)$.

Suppose conversely that $h_{n}(E) \backslash K \neq \varnothing$ and let $m_{0} \in h_{n}(E) \backslash K$. Let $S$ be an $(n-1)$-tuple over $A$ such that $m_{0} \in V(S) \backslash h(E \cap V(S))$. Consequently there exists a function $f \in A_{V(S)}$, such that $\left|f\left(m_{0}\right)\right|>$ $\max _{E \cap V(S)}|f(m)|$. Without loss of generality we can assume that $f$ does not vanish on $\operatorname{sp} A$. For $g=1 / f \in A^{-1}$ we have $\left|g\left(m_{0}\right)\right|<r=$ $\min _{E \cap V(S)}|g(m)|$. For any positive $\varepsilon<r$ we can find a neighborhood $V_{\varepsilon}$ of the set $E \cap V(S)$ in $E$ on which $|g(m)|>r-\varepsilon$. Hence for every $m \in E$ we have

$$
\left(C_{\varepsilon}^{2} \sum_{j=1}^{n-1}\left|f_{j}(m)\right|^{2}+|g(m)|^{2}\right)^{1 / 2}>r-\varepsilon
$$

for some positive constant $C_{\varepsilon}$ large enough. (5) holds also on $h_{n}(E)$ because the $n$-tuple $\left(C_{\varepsilon} f_{1}, \ldots, C_{\varepsilon} f_{n-1}, g\right)$ does not vanish on $h_{n}(E)$. In particular at $m_{0}$ we have $\left|g\left(m_{0}\right)\right|>r-\varepsilon$ and henceforth $\left\|g\left(m_{0}\right)\right\| \geq r$ because of the liberty of the choice of $\varepsilon$. Since this contradicts with the initial inequality $\left|g\left(m_{0}\right)\right|<r$, we conclude that $h_{n}(E) \subset K$. The theorem is proved. 
The sets described in Theorem 2 are precisely the hulls which were considered by Basener in [2] and used by him in his study of $q$ holomorphic functions.

THEOREM 3. Let $E$ be a closed subset of $\operatorname{sp} A$. Then $E \subset \cdots \subset$ $h_{n+1}(E) \subset h_{n}(E) \subset \cdots \subset h_{1}(E)=h(E) \subset \operatorname{sp} A$ and $\bigcap_{n \geq 1} h_{n}(E)=E$.

Proof. The first part of the statement is obvious because if (3) is fulfilled for every $(n+1)$-tuple $F$ of functions from $\mathrm{A}$ which does not vanish on $K$, then it is fulfilled also for every $n$-tuple which does not vanish on $K$.

The inclusion $\bigcap_{n} h_{n}(E) \supset E$ is clear. If $m_{0} \notin E$ then for every $m$ in $E$ we can find a function $f_{m} \in A^{-1}$ such that $\left|f_{m}(m)\right|>1$ and $\left|f_{m}\left(m_{0}\right)\right|<1$. Let $U_{m}$ be an open neighborhood of $m$ such that $\left|f_{m}(x)\right|>1$ for any $x \in U_{m}$. By a compactness argument there exist finitely many points $m_{1}, \ldots, m_{k}$ in $E$ such that $E \subset U_{m_{1}} \cup$ $\cdots \cup U_{m_{k}}$. By replacing each $f_{m_{j}}$ by some of its power we can assume from the beginning that $\left|f_{m_{j}}\left(m_{0}\right)\right|<1 / k$ and $\left|f_{m_{j}}(x)\right|>1$ on $U_{m_{j}}$. Hence for $F=\left(f_{m_{1}}, \ldots, f_{m_{k}}\right) \in A^{k}$ we have that $\|F(m)\| \neq 0$ on $\operatorname{sp} A,\|F(m)\|>1$ on $E$ and $\left\|F\left(m_{0}\right)\right\|<1$. Hence $m_{0} \notin h_{k}(E)$ and moreover $m_{0} \notin \bigcap_{n} h_{n}(E)$. We conclude that $\bigcap_{n} h_{n}(E) \subset E$, as required.

\section{Multi-tuple Shilov boundaries and multi-tuple hulls.}

Definition 3 (Basener, Sibony). The $n$-tuple Shilov boundary $\partial^{(n)} A$ of a commutative Banach algebra $A$ is the following subset of $\operatorname{sp} A$ :

$$
\partial^{(n)} A=\left[\bigcup\left\{\partial A_{V\left(f_{1}, \ldots, f_{n-1}\right)}:\left(f_{1}, \ldots, f_{n-1}\right) \in A^{n-1}\right\}\right],
$$

where $A^{0}=\{0\}$ (see [1], [6], also [13]).

It is easy to check that $\partial A=\partial^{(1)} A \subset \partial^{(2)} A \subset \partial^{(3)} A \subset \cdots \subset \partial^{(n)} A \subset$ $\cdots \subset \operatorname{sp} A$. We shall recall some of the basic properties of multi-tuple Shilov boundaries.

THEOREM 4 ([10, Theorem 1]). $\partial^{(n)} A$ is the smallest closed subset of $\operatorname{sp} A$ on which every regular $n$-tuple $F=\left(f_{1}, \ldots, f_{n}\right) \in A$ assumes the minimum of its norm

$$
\|F(m)\|=\left(\sum_{j=1}^{n}\left\|\hat{f}_{j}(m)\right\|^{2}\right)^{\frac{1}{2}}, \quad m \in \operatorname{sp} A .
$$


In other words Theorem 4 says that $\partial^{(n)} A$ is the smallest closed subset of $\operatorname{sp} A$ on which every regular $n$-tuple $F=\left(f_{1}, \ldots, f_{n}\right) \in A$ assumes the minimum of its norm (6).

Theorem 5 ([10, Theorem 3]). $\partial^{(n)} A$ is the smallest closed subset of $\operatorname{sp} A$ such that the inclusion

$$
F\left(\partial^{(n)} A\right) \supset b F(\operatorname{sp} A)
$$

holds for every $n$-tuple $F=\left(f_{1}, \ldots, f_{n}\right) \in A^{n}$.

The next proposition in particular says that, in the case of algebra $A=A\left(B^{n}(1)\right), r_{k}\left(S^{n}(1)\right)=B^{n}(1)$ for any $k<n$ unlike the case $k=n$ when, according to Proposition $3, S^{n}(1)$ is $n$-tuple rationally $A$-convex and hence $r_{n}\left(S^{n}(1)\right)=S^{n}(1)$.

Proposition 6. $r_{k}\left(\partial^{(n)} A\right)=\operatorname{sp} A$ for each $k<n$; if $r_{n}\left(\partial^{(n)} A\right)=$ $\operatorname{sp} A$ then $r_{n}(E) \neq \operatorname{sp} A$ for every proper closed subset $E$ of $\partial^{(n)} A$.

Indeed, as shown in [10], $V(G) \cap \partial^{(n)} A \neq \varnothing$ for every irregular $k$-tuple $G \in A^{k}, 1 \leq k \leq n-1$. From Proposition 2 we conclude that $r_{k}\left(\partial^{(n)} A\right)=\operatorname{sp} A$ for every $k<n$. If $E \neq \partial^{(n)} A$ then there is an $n$-tuple $F \in A^{n}$ with $\min _{m \in E}\|F(m)\|>\min _{m \in \operatorname{sp} A}\|F(m)\|=0$ and consequently, by Definition $1, r_{n}(E) \neq r_{n}\left(\partial^{(n)} A\right)=\operatorname{sp} A$.

Proposition 7. $h_{n}(E)=\operatorname{sp} A$ if and only if $E$ contains the $n$-tuple Shilov boundary $\partial^{(n)} A$ of $A$.

Indeed, from Definition 2 and Theorem 4 it follows that $h_{n}\left(\partial^{(n)} A\right)$ $=\operatorname{sp} A$. Theorem 4 shows that $h_{n}(E) \neq \operatorname{sp} A$ if $E \backslash \partial^{(n)} A \neq \varnothing$.

Given an $n$-tuple $\left(f_{1}, \ldots, f_{n}\right) \in A^{n}$ let $\sigma\left(f_{1}, \ldots, f_{n}\right)$ be the joint spectrum of the $n$-tuple $\left(f_{1}, \ldots, f_{n}\right)$, i.e.

$$
\sigma\left(f_{1}, \ldots, f_{n}\right)=\left\{\left(f_{1}(m), \ldots, f_{n}(m)\right): m \in \operatorname{sp} A\right\} .
$$

Proposition 8. The joint spectrum of every n-tuple $F$ over $A$ is contained in the n-tuple polynomial hull of the set $F\left(\partial^{(n)} A\right)$, i.e.

$$
\sigma(F)=F(\operatorname{sp} A) \subset \pi_{n}\left(F\left(\partial^{(n)} A\right)\right), \quad F \in A^{n} .
$$

Proof (see also [11]). Since by the Theorem 4

$$
\min _{m \in \partial^{(n)} A}\|G(m)\|=\min _{m \in \operatorname{sp} A}\|G(m)\|
$$


for every regular $n$-tuple $G \in A^{n}$, the equality

$$
\begin{aligned}
\min _{m \in \partial^{(n)} A} & \left\|\left(p_{1} \circ F(m), \ldots, p_{n} \circ F(m)\right)\right\| \\
= & \min _{m \in \operatorname{sp} A}\left\|\left(p_{1} \circ F(m), \ldots, p_{n} \circ F(m)\right)\right\|
\end{aligned}
$$

and, equivalently,

$$
\min _{m \in F\left(\partial^{(n)} A\right)}\left\|\left(p_{1}, \ldots, p_{n}\right)(\mathbf{z})\right\|=\min _{m \in F(\mathbf{s p} A)}\left\|\left(p_{1}, \ldots, p_{n}\right)(\mathbf{z})\right\|
$$

are fulfilled for every $n$-tuple of polynomials $p_{1}, \ldots, p_{n}$ in $\mathbf{C}^{n}$ without joint zeros on $\sigma(F)=F(\operatorname{sp} A)$. Definition 2 indicates that the sets $F\left(\partial^{(n)} A\right)$ and $\sigma(F)$ have equal $n$-tuple polynomial hulls and consequently $\sigma(F) \subset \pi_{n}\left(F\left(\partial^{(n)} A\right)\right)$, as claimed.

A well known theorem from the uniform algebra theory says that if an algebra is generated (linearly) by its subset $\Lambda$, then the range of its Shilov boundary $\partial A$ via the spectral mapping $\widehat{\Lambda}: \operatorname{sp} A \rightarrow \mathbf{C}^{\Lambda}$ : $m \longmapsto\{f(m): f \in \Lambda\}$ of $\Lambda$ is the smallest closed subset of $\mathbf{C}^{\Lambda}$ whose polynomial hull is equal to the polynomial hull of the set $\widehat{\Lambda}(\operatorname{sp} A)$. In the next theorem we make use of the $n$-tuple $A$-convex boundaries in order to obtain an extension of this result for the multi-tuple Shilov boundaries $\partial^{(n)} A$. Namely

THEOREM 6. Let $S=\left\{b_{\lambda}\right\}_{\lambda \in \Lambda}$ be a set which generates linearly a uniform algebra $A$. Then the range $\widehat{\Lambda}\left(\partial^{(n)} A\right)$ of the $n$-tuple Shilov boundary via $\widehat{\Lambda}$ is the smallest among all compact subsets $E$ in $\mathbf{C}^{\Lambda}$ whose $n$-tuple polynomial hulls $\pi_{n}(E)$ are equal to the $n$-tuple polynomial hull $\pi_{n}(\widehat{\Lambda}(\operatorname{sp} A))$ of the range of $\widehat{\Lambda}$.

Proof (see also [11]). Without loss of generality we can assume that $E$ is a subset of $\widehat{\Lambda}(\operatorname{sp} A)$ and consequently that $E=\widehat{\Lambda}(K)$ for some compact set $K \in \operatorname{sp} A$. The $n$-tuple polynomial hulls $\pi_{n}(E)=$ $\pi_{n}(\widehat{\Lambda}(K))$ and $\pi_{n}(\widehat{\Lambda}(\operatorname{sp} A))$ are equal if and only if $\min _{\mathbf{z} \in \widehat{\Lambda}(K)}\|P(\mathbf{z})\|=$ $\min _{\mathrm{z} \in \widehat{\Lambda}(\mathrm{sp} A)}\|P(\mathbf{z})\|$ for every $n$-tuple $P=\left(p_{1}, \ldots, p_{n}\right)$ of polynomials in $\mathbf{C}^{\Lambda}$ with $\|P(\mathbf{z})\| \neq 0$ on $\pi_{n}(\widehat{\Lambda}(\operatorname{sp} A))$. Equivalently, $\pi_{n}(\widehat{\Lambda}(K))=$ $\pi_{n}(\widehat{\Lambda}(\operatorname{sp} A))$ if and only if $\min _{m \in K}\|P \circ \widehat{\Lambda}(m)\|=\min _{m \in \operatorname{sp} A}\|P \circ \widehat{\Lambda}(m)\|$ for any $n$-tuple of type $P \circ \widehat{\Lambda} \in A^{n}$ which does not vanish on the set $\widehat{\Lambda}^{-1}\left(h_{n}(\widehat{\Lambda}(\operatorname{sp} A))\right)=\operatorname{sp} A$. Since the set of functions $p_{j} \circ \widehat{\Lambda}(m)$ is dense in $\mathrm{A}, \pi_{n}(\widehat{\Lambda}(K))=\pi_{n}(\widehat{\Lambda}(\operatorname{sp} A))$ if and only if $\min _{m \in K}\|F(m)\|=$ $\min _{m \in \operatorname{sp~} A}\|F(m)\|$ for every regular $n$-tuple $F \in A^{n}$. By Theorem 
4 the $n$-tuple Shilov boundary $\partial^{(n)} A$ is the smallest closed subset of sp $A$ with the last property; and therefore $\widehat{\Lambda}\left(\partial^{(n)} A\right)$ is the smallest closed subset of $\mathbf{C}^{\Lambda}$ whose $n$-tuple polynomial hull is the same as the $n$-tuple polynomial hull of the set $\widehat{\Lambda}(\operatorname{sp} A))$, as claimed.

5. Remarks. Recall that function space is called any linear subspace of the space $C(X)$, where $X$ is a compact Hausdorff space, which is closed under the uniform norm $\|f(x)\|=\max _{x \in X}|f(x)|$, contains the constants and separates the points of $X$. It can be shown that for every function space $B$ over $X$ there exists a smallest set $\operatorname{Sh}_{B}(X)$ among all closed subsets $E$ of $X$ such that

$$
\min _{x \in X}|f(x)|=\min _{x \in E}|f(x)|
$$

for every nonvanishing on $X$ function $f$ in $B$. In general $\operatorname{Sh}_{B}(X)$ does not coincide with the usual Shilov boundary $\partial B$ of $B$ which, by definition is the smallest among all closed subsets $E$ of $X$ such that

$$
\max _{x \in X}|f(x)|=\max _{x \in E}|f(x)|
$$

for every function $f$ in $B$. The $n$-tuple Shilov boundary of a function space $B$ is the set

$$
\operatorname{Sh}_{B^{n}}(X)=\left[\bigcup \operatorname{Sh}_{B}(V(F)): F=\left(f_{1}, \ldots, f_{n-1}\right) \in B^{n-1}\right],
$$

where $V(F)=\left(f_{1}, \ldots, f_{n-1}\right)^{-1}(\mathbf{0})$.

It is a matter of a simple verification to check that all the above results hold not only for uniform algebras but for function spaces as well with the Shilov boundary $\partial A$ replaced with the boundary $\mathrm{Sh}_{B}(X)$ and the $n$-tuple Shilov boundary $\partial^{(n)} A$ replaced with the $n$-tuple Shilov boundary $\operatorname{Sh}_{B^{n}}(X)$.

The author is grateful to the referée for his or her useful suggestions.

\section{REFERENCES}

[1] R. Basener, A generalized Shilov boundary and analytic structure, Proc. Amer. Math. Soc., 47 (1975), 98-104.

[2] - Several dimensional properties of the spectrum of a uniform algebra, $\mathrm{Pa}-$ cific J. Math., 74 (1978), 297-306.

[3] G. Corach and F. Suárez, Generalized rational convexity in Banach algebras, Pacific J. Math., 140 (1989), 35-51.

[4] T. Gamelin, Uniform Algebras, Prentice-Hall Inc., Englewood Cliffs, New Jersey (1969).

[5] D. Kumagai, Subharmonic functions and uniform algebras, Proc. Amer. Math. Soc., 78 (1980), 23-29. 
[6] N. Sibony, Multi-dimensional analytic structure in the spectrum of a uniform algebra, Springer Lect. Notes in Math., 512 (1976), 139-175.

[7] Z. Slodkowski, Local maximum property and q-plurisubharmonic functions in uniform algebras, J. Math. Anal. Appl., 115 (1986), 105-130.

[8] - Analytic perturbations of the Taylor spectrum, Trans. Amer. Math. Soc., 297 (1986), 319-336.

[9] E. Stout, The Theory of Uniform Algebras, Bogden and Quigley, Tarrytown on Hudson (1971).

[10] T. Tonev, New relations between Sibony-Basener boundaries, Springer Lect. Notes in Math., 1277 (1987), 256-262.

[11] _ General Complex-Analytic Structures in Uniform Algebra Spectra, preprint (in Bulgarian), Sofia (1987), 300 pp.

[12] _ Multi-dimensional analytic structures and uniform algebras, Houston J. Math., 17 (1991), 101-108.

[13] J. Wermer, Banach Algebras and Several Complex Variables, Grad. Texts in Math., Springer Verlag (1976).

Received June 4, 1990 and in revised form January 17, 1991.

THE UNIVERSITY OF TOLEDO

TOLEDO, OH 43606-3390 



\section{PACIFIC JOURNAL OF MATHEMATICS EDITORS}

\author{
V. S. VARADARAJAN \\ (Managing Editor) \\ University of California \\ Los Angeles, CA 90024-1555-05 \\ Herbert Clemens \\ University of Utah \\ Salt Lake City, UT 84112 \\ THOMAS ENRIGHT \\ University of California, San Diego \\ La Jolla, CA 92093
}

Nicholas ERcolani

University of Arizona

Tucson, AZ 85721

R. FINN

Stanford University

Stanford, CA 94305

VAUGHAN F. R. JONES

University of California

Berkeley, CA 94720

STEVEN KeRCKHOFF

Stanford University

Stanford, CA 94305

\section{C. MOORE \\ University of California \\ Berkeley, CA 94720}

MARTIN SChaRLEMANN

University of California

Santa Barbara, CA 93106

HAROLd STARK

University of California, San Diego

La Jolla, CA 92093

\section{ASSOCIATE EDITORS}

\begin{tabular}{|c|c|c|c|c|}
\hline ARENS & $\begin{array}{l}\text { E. F. BECKENBACH } \\
(1906-1982)\end{array}$ & NeumanN & $\begin{array}{l}\text { F. Wolf } \\
(1904-1989)\end{array}$ & K. Yoshida \\
\hline \multicolumn{5}{|c|}{ SUPPORTING INSTITUTIONS } \\
\hline \multirow{2}{*}{\multicolumn{2}{|c|}{$\begin{array}{l}\text { IVERSITY OF ARIZONA } \\
\text { IIVERSITY OF BRITISH COLUMBIA }\end{array}$}} & UNIVERS & Y OF OREGON & \\
\hline & & UNIVERS & OF SOUTHER & IIA \\
\hline \multicolumn{2}{|c|}{ LIFORNIA INSTITUTE OF TECHNOLOGY } & STANFO & UNIVER & \\
\hline \multirow{2}{*}{\multicolumn{2}{|c|}{$\begin{array}{l}\text { IVERSITY OF CALIFORNIA } \\
\text { ONTANA STATE UNIVERSITY }\end{array}$}} & UNIVERS & OF HAWAII & \\
\hline \multirow{2}{*}{\multicolumn{2}{|c|}{$\begin{array}{l}\text { ONTANA STATE UNIVERSITY } \\
\text { IIVERSITY OF NEVADA, RENO }\end{array}$}} & UNIVERS & YF TOKYO & \\
\hline & & UNIVERS & I OF UTAH & \\
\hline \multirow{2}{*}{\multicolumn{2}{|c|}{$\begin{array}{l}\text { W MEXICO STATE UNIVERSITY } \\
\text { EGON STATE UNIVERSITY }\end{array}$}} & WASHING & N STATE UNI & SITY \\
\hline & & UNIVERS & NASI & \\
\hline
\end{tabular}

The Supporting Institutions listed above contribute to the cost of publication of this Journal, but they are not owners or publishers and have no responsibility for its content or policies.

Mathematical papers intended for publication in the Pacific Journal of Mathematics should be in typed form or offset-reproduced (not dittoed), double spaced with large margins. Please do not use built up fractions in the text of the manuscript. However, you may use them in the displayed equations. Underline Greek letters in red, German in green, and script in blue. The first paragraph must be capable of being used separately as a synopsis of the entire paper. In particular it should contain no bibliographic references. Please propose a heading for the odd numbered pages of less than 35 characters. Manuscripts, in triplicate, may be sent to any one of the editors. Please classify according to the 1991 Mathematics Subject Classification scheme which can be found in the December index volumes of Mathematical Reviews. Supply name and address of author to whom proofs should be sent. All other communications should be addressed to the managing editor, or Elaine Barth, University of California, Los Angeles, California 90024-1555-05.

There are page-charges associated with articles appearing in the Pacific Journal of Mathematics. These charges are expected to be paid by the author's University, Government Agency or Company. If the author or authors do not have access to such Institutional support these charges are waived. Single authors will receive 50 free reprints; joint authors will receive a total of 100 free reprints. Additional copies may be obtained at cost in multiples of 50 .

The Pacific Journal of Mathematics (ISSN 0030-8730) is published monthly except for July and August. Regular subscription rate: $\$ 190.00$ a year (10 issues). Special rate: $\$ 95.00$ a year to individual members of supporting institutions.

Subscriptions, orders for numbers issued in the last three calendar years, and changes of address should be sent to Pacific Journal of Mathematics, P.O. Box 969, Carmel Valley, CA 93924, U.S.A. Old back numbers obtainable from Kraus Periodicals Co., Route 100, Millwood, NY 10546.

The Pacific Journal of Mathematics at P.O. Box 969, Carmel Valley, CA 93924 (ISSN 0030-8730) is published monthly except for July and August. Second-class postage paid at Carmel Valley, Californiä 93924, and additional mailing offices. Postmaster: send address changes to Pacific Journal of Mathematics, P.O. Box 969, Carmel Valley, CA 93924.

\section{PUBLISHED BY PACIFIC JOURNAL OF MATHEMATICS, A NON-PROFIT CORPORATION}




\section{Pacific Journal of Mathematics}

\section{Vol. 151, No. $2 \quad$ December, 1991}

Michael G. Eastwood and A. M. Pilato, On the density of twistor

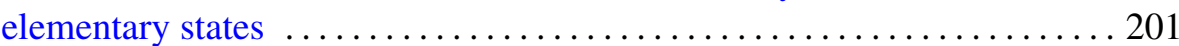

Brian E. Forrest, Arens regularity and discrete groups $\ldots \ldots \ldots \ldots \ldots \ldots 217$

Yu Li Fu, On Lipschitz stability for F.D.E ..................... 229

Douglas Austin Hensley, The largest digit in the continued fraction expansion of a rational number $\ldots \ldots \ldots \ldots \ldots \ldots \ldots \ldots \ldots \ldots \ldots . \ldots 237$

Uwe Kaiser, Link homotopy in $\mathbb{R}^{3}$ and $S^{3}$

Ronald Leslie Lipsman, The Penney-Fujiwara Plancherel formula for abelian symmetric spaces and completely solvable homogeneous

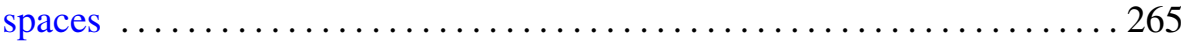

Florin G. Radulescu, Singularity of the radial subalgebra of $\mathscr{L}\left(F_{N}\right)$ and the

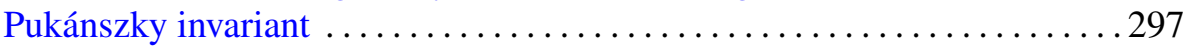

Albert Jeu-Liang Sheu, The structure of twisted SU(3) groups $\ldots . \ldots \ldots . \ldots 307$

Morwen Thistlethwaite, On the algebraic part of an alternating link . . . . . 317

Thomas (Toma) V. Tonev, Multi-tuple hulls .................. 335

Arno van den Essen, A note on Meisters and Olech's proof of the global asymptotic stability Jacobian conjecture ..................... 351

Hendrik J. van Maldeghem, A characterization of the finite Moufang hexagons by generalized homologies

Bun Wong, A note on homotopy complex surfaces with negative tangent bundles

Chung-Tao Yang, Any Blaschke manifold of the homotopy type of $\mathbf{C} P^{n}$ has the right volume 